\title{
Postulates for Linear Connections in Abstract Vector Spaces ${ }^{(1)}$.
}

By A. D. Michal (Pasadena, California, U. S. A.).

Introduction. - In recent years a large portion of the work in $n$-dimensional differential geometry $\left(^{2}\right)$ has been centered around the notion of a linear connection. My studies on infinitely many dimensional differential geometries $\left({ }^{3}\right)$ in functional spaces have led me to the introduction of functional linear connections. Early in the development of these infinitely many dimensional geometries it became clear that some of the main properties of linear connections could be abstracted in normed vector spaces. In 1933 definitive results were obtained along this direction and the first chapter in abstract differential geometry was written $\left({ }^{+}\right)$.

(1) Presented to the "American Math. Soc. », Dec. 1, 1933, under a slightly different title. Cf. «Bulletin of A. M.S. ", abstract n. ${ }^{\circ}$ 337, Vol. 39 (November, 1933).

(2) The recent Jiterature on finite dimensional differential geometry is extensive. We refer the reader to the following representative books on the subject. L. P. EINSENHART, Riemannian Geometry (1926), Non-Riemannian Geometry ("A. M. S. Coll. Publications", Vol. VIII, 1927), Continuous Groups of Transformations (1933); J. A. ScHouteN, Der RicciKalkul (1924); O. VEBLEN, Invariants of Quadratic Differential Forms (1927), Projective Relativitätstheorie ("Ergebnisse Der Mathematik Und Ihrer Grenzgebiete ", 1983); T. X. Thomas, Differential Invariants of Generalized Spaces (1934); DuscheK-MAYnR, Lehrbuch Der Differentialgeometrie (vol. I and II, 1930); T. LEv-CrviTA, The Absolute Differential Calculus (1927); E. CARTaN, Leçons su La Géométrie Des Espaces De Riemann (1928), La Theorie Des Groupes Finis Et Continus Et L'Anrlysis Situs (* Mémorial des Sciences Ma* thématiques", fasc. 42, 1930); G. VxтAL, Geometria Nello Spazio Hilbertiano (1929); W. BrAschke, Differentialgeometrie (vol. IT, 1928); H. WEY, Space-Time-Matter ("English translation", 1921); O. $\mathrm{VEBLEN}_{\mathrm{ELN}}$ and J. H. C. WHITEHEAD, The Foundations of Differential Geometry (1932); D. J. STruIK. Theory of Linear Connections ("Ergebnisse Der Mathematik Und Thre: Grenzgebiete ", 1934); V. Hlavaty, Les Courbes De La Variété Générale à n Di mensions ("Mémorial des Sciences Mathématiques », Fasc. 63, 1934).

(3) A. D. Micnal, "American Journal of Math. ", vol. 50 (1928), pp. 473517. This paper was previously presented to the American Math. Soc, at the New York meeting, Oetober 1927 (Cf. "Bulletin of A. M. S. ", vol. 34, Jan. 1928, pp. 8-9 for the abstracts). Following the publication of this initial paper on the subject there appeared numerous papers on functional differential geometry and related topies by F. Confonto, A. Kaw AGUCHI; M. Kerner, A. D. Michal, G. C. Moisil, T. S. Pe rerson and H. P. Thielman.

(4) A. D. Mrchax, «Bull. of American Math. Soe. ", Vol. 39 (1983), pp. 879.881. 
Since then several new theorems on differentials and total differential equations in abstract vector spaces were proved and were helpful in a drastic reduction of the postulates as well as in deriving new theorems on abstract linear connections.

Before we proceed with a brief description of the contents of the various paragraphs of our paper we shall make a few remarks on the reasons for choosing normed vector spaces as manifolds and on the nature of an abstract differential geometry. From the work of E. CARTAN and others it has become clear that a differential geometry is concerned not with one space but with several spaces and their interconnections by means of interspace functional transformations. One of the spaces is often called the underlying manifold and the remaining spaces, the associated manifolds. We take the point of view that the universe of discourse of a differential geometry consists of a collection of spaces and a set of inter-space functional transformations at least one of which possesses a differential.

Moreover the differentiability of at least one of these transformations plays an essential role in the theory. In actual practice some of these interspace functional transformations are only required to induce differentiable transformations between maps of the spaces. The maps are usually instances of abstract normed vector spaces such as number spaces and functional spaces.

As far as the author has been able to ascertain, the most general spaces over which uique differentials can be defined are normed vector spaces (1) (BANACH spaces are normed vector spaces with a real number system as the multiplier number system). Differentials can be defined in more general spaces but we have not been able to prove that the differential of a given function in such general spaces is unique. Because of the above reasons it appears that normed vector spaces are of fundamental importance for the differential geometer. The degree of generality of normed rector spaces is however considerable, for it must be remembered that abstract HruBerT space ( $\left.{ }^{3}\right)$ is a special kind of a normed vector space and that most of the functional spaces (") of analysis are instances of an abstract normed vector space.

(1) Numerous references to papers on abstract space theory can be found in M. Frit. CHET, Les Espaces Abstraits (1928), and in S. BANACH, Theorie Des Opérations Linéares (1932).

(') M. H. STоNк, Lineas Transformations in Hilbert Space ("A. M. S. Colloquium publications ", 1932), where references are given to HuberT's, Ruesz's and v. NeumanN's papers.

(3) For the theory of functionals and related subjects see G. C. Evans, Fumctionals And Their Applications ("A. M. S. colloquium publications", 1918); V. Volterra, Theory of Functionals (1930); P. LÉYY, Analyse Fonctionnelle (1922). See also FrÉcHET's and BANACH's books. 
The first paragraph contains a few preliminary theorems on the differentials of functions depending linearly on one or more abstract parameters. In particular a fundamental theorem is proved on the total differential of the inverse of a solvable linear function $f(x, x)$ (See Theorem 1.4).

In $\S 2$ we give six postulates, in addition to the vector space postulates, for a linear connection and we present a functional instance for their consistency. It is worthwhile to mention here that as a rule we abstract the forms rather than the coefficients of forms. A notable exception to this general procedure is furuished by the postulates for a normed vectorial ring given in $\S 6$. On the basis of the postulates of $\S 2$ we are enabled to give in $\$ 3$ the definition of a curvature form (curvature for brevity). Theorem 3.7 of $\$ 3$ and Theorems 4.1 and 4.2 of $\S 4$ deal with solvable linear functions that satisfy abstract total differential equations. We hope to make clear elsewhere the fundamental nature of these theorems in connection with the differential geometry of the group manifold of continuous transformation groups with abstract parameters. A brief treatment of parallelism of abstract vector fields with respect to a given curve in the underlying vector manifold is given in $\$ 5$.

A set of postulates is given in $\$ 6$ with a special end in view. A vector space $E_{2}$, a normed vectorial ring, is introduced and amongst the postulated functional operations is a class of linear functions, called contractions, on $E_{2}$ to the real numbers. On the basis of these postulates we make a definition of a class of «generalized RIocr» forms. The functional instance presented for the consistency of the postulates contains a continuous infinitude of contractions and hence a continuous infinitude of «generalized Ricci» forms.

Finally in $\& 7$ postulates are given for a second linear connection in addition to the postulates of $\S 2$ for the first linear connection. The funda. mental identity relating the two curvatures is contained in Theorem 7.2.

The author has initiated elsewhere (1) the study of "Riemannian » differential geometries in abstract vector spaces. The postulates for such a geometry lead to two abstract "CHRISTOFFEL symbols of the second kind » that are special linear connections of the kind treated in $\$ 2$ and $\xi 7$ of the present paper.

\$ 1. Preliminary theorems on Fréchet differentials. - We shall give in this paragraph a few new theorems on FRECHET differentials in abstract

(1) A. D. MichaL, "Bull. of American Math. Soc. ", vol. 39 (1933), abstracts 382 and 333 , p. 879 . 
vector spaces that will be needed in the development of our subject. By an abstract vector space (vector space for short) we mean a complete normed linear abstract space closed under multiplication by real numbers. Such spaces are often called BANACH spaces in the literature.

There is a real advantage gained in using the same symbol for a norm $\|\ldots\|$ in the simultaneous consideration of several vector spaces. Similar remarks hold good for the symbols $t$, 0 , and $=$ in the simultaneous consideration of real numbers and vector spaces. We shall adhere to these simplifications in notation throughout our paper. There need not be any confusion in these matters as the context will make clear the meaning of the symbols used.

We begin with the

Theonem 1.1. - Let $\mathrm{E}_{1}, \mathrm{E}_{2}$ and $\mathrm{E}_{3}$ be three vector spaces. If $\mathrm{F}(\mathrm{x}, \alpha)$ is a function (") on $\left.\mathrm{E}_{1}\left(\left(\mathrm{x}_{1}\right)\right)_{\mathrm{a}}\right) \mathrm{E}_{2}$ to $\mathrm{E}_{3}$, linear (") in $\alpha$ and possessing a partial differential $\mathrm{F}(\mathrm{x}, \alpha ; \delta \mathrm{x})$ that is continuous in $\mathrm{x}$, then the total differential $\mathrm{F}(\mathrm{x}, \alpha ; \delta \mathrm{x}, \delta \alpha)$ exists, $\left({ }^{3}\right)$ is continuous in $\mathrm{x}, \alpha, \delta \mathrm{x}, \delta \alpha$, and is given by

$$
F(x, \alpha ; \delta x, \delta \alpha)=F(x, \alpha ; \delta x)+F(x, \delta \alpha) .
$$

Proof:

$$
\begin{gathered}
\|F(x+\delta x, \alpha+\delta \alpha)-F(x, \alpha)-F(x, \alpha ; \delta x)-F(x, \delta \alpha)\| \\
\leq \| F(x+\delta x, \alpha)-F(x, \alpha)-F(x, \alpha ; \delta x\|+\| F(x+\delta x, \delta \alpha)-F(x, \delta \alpha) \| .
\end{gathered}
$$

From our hypotheses on $F(x, \alpha ; \delta x)$ and a few results of M., KERNER ( $\left.{ }^{4}\right)$

(1) All functions considered in this paper will be, without explicit mention, single valued. We use E. H. Moork's convenient terminology \& $\Phi\left(x_{1}, x_{2}, \ldots, x_{q_{t}}\right)$ is a function on $c_{1} c_{2} \ldots c_{n}$ to $c$, to denote that $\Phi$ is a funetion whose ith argument ranges over a class of elements $c_{i}$ and whose values are elements in the class $c$. We shall also use the symbol $c_{i}(s)$ to denote that the ith argument of the function $\Phi$ ranges over the subset $s$ of the class $c_{i}$. The classes $c_{i}$ will be usually vector spaces and the subsets $s$ will usually be open neighborhoods $\left(x_{0}\right)_{x}$, i. e, the set of elements $x$ that satisfy the inequality $\left\|x-x_{0}\right\|<a$ for a fixed element $x_{0}$ and fixed positive numbsr $a$. Most of the theorems in the paper continue to hold if more general connected sets $s$ are used in the place of $\left(x_{0}\right)_{\alpha}$. Without any further statement we shall restrict ourselves to functions fow which the substitution of equal elements is a valid operation.

$\left({ }^{2}\right)$ Additive and continuons (and hence homogeneous of degree one).

(3) In order to do away with long eircumlocutions we shall understand throughout our paper, unless a statement to the contrary is made, that statements on properties of a function will hold good for all values of the arguments lying in the domains of definition of the function and that assertions on properties of differentials of a function will hold good for all such values of the arguments of the given function and for all values of the new arguments that the differential introduces. For example, the assertions for $F(x, \alpha ; \varepsilon x, \delta x)$ in Theorem 1.1 are understood to hold for all $x$ in $\left(x_{0}\right)_{a}$, all $x$ in $E_{z}$, all $\partial x$ in $E_{1}$, and all $\delta x$ in $E_{2}$.

(4) "Annals of Math. *, vol. 34 (1933), pp. 548-552. 
we obtain the inequalities

$$
\|F(x+\delta x, \delta \alpha)-F(x, \delta \alpha)\| \leq \int_{0}^{1}\|F(x+s \delta x, \delta \alpha ; \delta x)\| d s
$$

and $(1)$

$$
\int_{0}^{l}\|F(x+s \delta x, \delta \alpha ; \delta x)\| d s \leq m\|\delta x\|\|\delta \alpha\| .
$$

The inequality (1.3) holds for $\|\delta x\|$ sufficiently small and $m$ is a fixed positive number. Hence given an $\varepsilon>0$, there exists a $\rho_{1}>0$ and a $\rho_{2}>0$ such that

$$
\|F(x+\delta x, \alpha)-F(x, \alpha)-F(x, \alpha ; \delta x)\| \leq \varepsilon_{2}^{\varepsilon}\|\delta x\| \text { for }\|\delta x\|<\rho_{1}
$$

and

$$
\int_{0}^{1}\|F(x+s \delta x, \delta \alpha ; \delta x)\| d s \leq \frac{\varepsilon}{2}\|\delta \alpha\| \text { for }\|\delta x\|<\rho_{2} .
$$

Let $\rho$ be the smaller of $\rho_{1}$, and $\rho_{2}$. From the above inequalities we obtain

$$
\|F(x+\delta x, \alpha+\delta \alpha)-F(x, \alpha)-F(x, \alpha ; \delta x)-F(x, \delta \alpha)\| \leq \frac{\varepsilon}{2}(\|\delta x\| 1-\|\delta \alpha\|)
$$

for $\|\delta x\|<\rho$ and all $\delta \alpha$. Since for arbitrary $\delta x$, $\delta \alpha$ (greater of $\|\delta x\|$ and $\|\delta \alpha\|) \equiv\|\delta x, \delta \alpha\| \geq \frac{1}{2}(\|\delta x\|+\|\delta \alpha\|)$ we see that

$$
\begin{gathered}
\|F(x+\delta x, \alpha+\delta \alpha)-F(x, \alpha)-F(x, \alpha ; \delta x)-F(x, \delta \alpha)\| \leq \varepsilon\|\delta x, \delta \alpha\| \\
\text { for }\|\delta x, \delta \alpha\|<\rho .
\end{gathered}
$$

But clearly $F(x, \alpha ; \delta x)+F(x, \delta \alpha)$ is continuous and additive in $\delta x, \delta \alpha$. Hence the last inequality shows that $F(x, \alpha ; \delta x, \delta \alpha)$ exists and is given by (1.1). Moreover the continuity of $F(x, \alpha ; \delta x, \delta \alpha)$ in $x, \alpha, \delta x$, $\delta \alpha$ is obtained readily by observing that $F(x, \alpha ; \delta x)$ is linear in $\alpha$, linear in $\delta x$, and that $F(x, \delta \alpha)$ is linear in $\delta \alpha$.

(1) It is well known that if the Fisches differential of a function exists then the Gateaux differential also exists and the two are equal. Hence if a function $F(x, x)$ is linear in $\alpha$, one can show readily with the aid of a theorem of BANACH (see S. BANACH, " Fundamenta Mathematicae ", vol. 3 (1922), p. 157) that the Gateaux differential $\lim _{\lambda \rightarrow 0} \frac{E(\alpha+\lambda \delta x, \alpha)-E(x, \alpha)}{\lambda}$, if it exists, is linear in $\alpha$. Similarly for the FRÉcher differential $F(x, \alpha ; \varepsilon x)$. 
202 A. D. Mrchax: Postulates for Linear Connections in Abstract Vector Spaces

The following more general theorem can be proved in a similar fashion.

Theorem 1.2. - Let $\mathrm{E}_{1}, \mathrm{E}_{2}, \ldots, \mathrm{E}_{\mathrm{n}}, \mathrm{E}$ be $\mathrm{n}+1$ vector spaces. If $\mathrm{F}\left(\mathrm{x}, \alpha_{1}\right.$, $\left.\alpha_{2}, \ldots, \alpha_{\mathrm{n}-1}\right)$ is a function on $\mathrm{E}_{1}\left(\left(\mathrm{x}_{0}\right)_{\mathrm{a}}\right) \mathrm{E}_{z} \ldots \mathrm{E}_{\mathrm{n}}$ to $\mathrm{E}$, multitinear in $\alpha_{1}, \alpha_{2}, \ldots, \alpha_{\mathrm{n}-1}$ and possessing $\alpha$ partial differential $\mathrm{F}\left(\mathrm{x}, \alpha_{1}, \alpha_{2}, \ldots, \alpha_{n-1} ; \delta \mathrm{x}\right)$ that is continuous in $\mathrm{x}$, then the partial differentials $\mathrm{F}\left(\mathrm{x}, \alpha_{1}, \ldots, \alpha_{\mathrm{i}}, \ldots, \alpha_{\mathrm{n}-1} ; \delta x, \delta \alpha_{5}\right)$ $(\mathrm{i}=1,2, \ldots, \mathrm{n}-1)$ exist, are continuous (jointly) in all their variables, and are given by

(1.4) $F\left(x, \alpha_{1}, \ldots, \alpha_{i}, \ldots, \alpha_{n-1}, \delta x, \delta \alpha_{i}\right)=F\left(x, \alpha_{1}, \ldots, \alpha_{n-1} ; \delta x\right)+F\left(x, \alpha_{1}, \ldots, \delta \alpha_{i}, \ldots, \alpha_{n-1}\right)$.

We next prove.

Theorem 1.3. - Let $\mathrm{E}_{1}, \mathrm{E}_{2}, \mathrm{E}_{3}$ be three vector spaces and let $\mathrm{F}(\mathrm{x}, \alpha)$ be a function on $\mathrm{E}_{1}\left(\left(\mathrm{x}_{0}\right)_{\mathrm{a}}\right) \mathrm{E}_{2}$ to $\mathrm{E}_{3}$ with the following properties:

1) $\mathrm{F}(\mathrm{x}, \alpha)$ is linear in $\alpha$.

2) The first partial differential $\mathrm{F}\left(\mathrm{x}, \alpha ; \delta_{1} \mathrm{x}\right)$ exists.

3) The second partial differential $\mathrm{F}\left(\mathrm{x}, \alpha ; \delta_{1} \mathrm{x} ; \delta_{2} \mathrm{x}\right)$ exists and is continuous in $\mathrm{x}$.

Then the differential $\mathrm{F}(\mathrm{x}, \alpha ; \delta \mathrm{x}, \delta \alpha)$ exists and is continuous in $\mathrm{x}, \alpha, \delta \mathrm{x}, \delta \alpha$; and the second differential $\mathrm{F}\left(\mathrm{x}, \alpha ; \delta_{1} \mathrm{x} ; \delta_{2} \mathrm{x}, \delta \alpha\right)$ exists, is continuous in $\mathrm{x}, \alpha$, $\delta_{1} \mathrm{x}, \delta_{2} \mathrm{x}, \delta \alpha$, and is given by

$$
F\left(x, \alpha ; \delta_{1} x ; \delta_{2} x, \delta \alpha\right)=F\left(x, \alpha ; \delta_{1} x ; \delta_{2} x\right)+F\left(x, \delta \alpha ; \delta_{1} x\right) .
$$

Proof: From the existence of the second differential $F\left(x, \alpha ; \delta_{1} x ; \delta_{2} x\right)$ it follows that $F(x, \alpha ; \delta, x)$ is continuous in $x$. The hypotheses of Theorem 1.1 are thus satisfied. An application of Theorem 1.1 shows the validity of the first part of our theorem. To prove the second part, we first observe that $F\left(x, \alpha ; \delta_{1} x\right)$ is bilinear in $\alpha$ and $\delta_{1} x$. An application of Theorem 1.2 to $F\left(x, \alpha ; \delta_{1} x\right)$ completes the proof of the theorem.

An extension of Theorem 1.3 to higher differentials can be given.

In ordre that there will be no misunderstanding in the later paragraphs, we shall give here a definition of a solvable linear function.

Definition of a solvable linear function. Let $E_{1}, E_{2}, E_{3}$ be vector spaces. A function $f(x, \alpha)$ on $E_{1}\left(\left(x_{0}\right)_{a}\right) E_{2}$ to $E_{3}$, linear in $\alpha$, is said to be solvable linear function, if there exists a function $\bar{f}(x, \beta)$ on $E_{1}\left(\left(x_{0}\right)_{a}\right) E_{3}$ to $E_{2}$ (called the inverse function) such that

$$
\left\{\begin{array}{l}
f(x, \bar{f}(x, \beta))=\beta \\
\bar{f}(x, f(x, \alpha))=\alpha
\end{array}\right.
$$


Clearly the inverse function $\vec{f}(x, \beta)$ is unique and by a theorem of BanacI (1) and Schauder ( $\left.{ }^{2}\right)$ it is linear in $\beta$. Thus $f(x, \alpha)$ is the inverse function of $\vec{f}(x, \beta)$ and so $f(x, \beta)$ is itself a solvable linear function.

THEOREM 1.4 - Let $\mathrm{E}_{1}, \mathrm{E}_{2}, \mathrm{E}_{3}$ be vector spaces. If $\mathrm{f}(\mathrm{x}, \alpha)$ is a solvable linear function on $\mathrm{E}_{1}\left(\left(\mathrm{x}_{0}\right)_{a}\right) \mathrm{E}_{2}$ to $\mathrm{E}_{3}$ such that the first partial differential $\mathrm{f}(\mathrm{x}, \alpha ; \delta \mathrm{x})$ exists and is continuous in $\mathrm{x}$, then the inverse function $\overline{\mathrm{f}}(\mathrm{x}, \beta)$ possesses a first partial differential $\overline{\mathrm{f}}(\mathrm{x}, \beta ; \delta \mathrm{x})$ that is continuous in $\mathrm{x}$ and linear in $\beta$.

Proof: From our hypotheses it follows with the aid of a theorem proved elsewhere $\left({ }^{3}\right)$ that $\bar{f}(x, \beta ; \delta x)$ exists and is given by

$$
\vec{f}(x, \beta ; \delta x)=-\vec{f}(x, f(x, \vec{f}(x, \beta) ; \delta x)) .
$$

Clearly $f(x, \alpha ; \delta x)$ is linear in $\alpha$. Hence the theorem follows from well known results on the continuity of functions of functions (i) and a theorem of KerNer $(\%$.

8 2. Postulates for a linear connection. - - In this paragraph we give a set of postulates for a linear connection. Some of the consequences of these postulates are developed sufficiently so as to bring ont the curvature expression and some of its fundamental properties.

We deal with two vector spaces: $E$, the underlying manifold of our geometry, and $E_{1}$, an associated manifold of elements that will sometimes be called, for historical reasons, "contravariant vectors ». The following notations will be used in this paragraph unless otherwise indioated.

$$
\begin{aligned}
& x, x_{1}, x_{2}, \ldots, y, y_{1}, y_{2}, \ldots, \approx, z_{1}, z_{2}, \ldots \text { : variables ranging over } E \\
& \text { Capital Latin and all Greek letters: 》 } \gg E_{1} \\
& \text { Remaining lower case Latin letters: } » \gg R \text {, }
\end{aligned}
$$

the real number system.

Postulate 1. There exists a function $\Gamma(x, V, y)$, called a linear connection, on $E\left(\left(x_{11}\right)_{a}\right) E_{1} E$ to $E_{1}$, where $x_{4}$ is a fixed element of $E$ and $a$ is a fixed positive number.

(1) S. Banach, "Studia Mathematica », vol. 1 (1929). p. 238.

() J. Schauder, «tudia Mathematica », rol. 2 (1930), pp. 1.6.

(3) A. D. Mighal and V. Erconin, "Bull. of American Math. Soc.", vol. 40 (1984), abstracts 228, p. 580 and 386, pp. $814-815$. To be published in full elsewhere.

(4) M. Frécret, "Annales So. Ec. Normale s, vol. 12 (1925). See also T. Hrudrbranut and L. Graves, "Trans. of A. M. S.", vol. 29 (1927).

(5) M. KerNer, "Annals of Math. ", loe, cit. 
Postulate 2. $\Gamma\left(x, V_{1}+V_{2}, y\right)=\Gamma\left(x, V_{1}, y\right)+\mathrm{\Gamma}\left(x, V_{2}, y\right)$.

Postulate 3. $\Gamma\left(x, V, y_{1}+y_{2}\right)=\Gamma\left(x, V, y_{1}\right)+\Gamma\left(x, V, y_{2}\right)$.

Postulate $x^{x}\|\mathrm{\Gamma}(x, V, y)\| \leq A\|V\|\|y\|$, where $A$ is a fixed positive number.

Postulate 5. The first partial differential $\Gamma(x, V, y ; \delta x)$ exists.

Postulate $6 . \Gamma(x, V, y ; \delta x)$ is continuous in $x$.

Clearly these postulates together with the postulates for the vector spaces $E$ and $E_{\mathrm{x}}$ form a consistent system. This can be seen most easily by taking an instance in which the vector spaces $E$ and $E_{1}$ are both the real number space with the absolute value as norm. A logical analysis of the above six postulates together with the postulates for the vector spaces $E$ and $E_{1}$ will not be given here. The postulates as they stand above are not enumerated in a form suitable for independence considerations. They have however the advantage of simplicity and perspicuity over the postulate system that is stated for such a purpose.

The reader will have no difficulty in giving non-trivial instances of our postulates from the realm of $n$-dimensional differential geometries (i) as well as from the author's $\left({ }^{2}\right)$ infinitely many dimensional differential geometries.

We give here briefly one of the many new instances of our postulates. Let the vector space $E$ be the well known "space of continnous functions 》 $x^{a}$. Before going any further in this instance we are forced to make a digression and make clear a few matters of notation. We shall write continuous real variables as indices and shall denote a RIEMANN integration over the whole range of an index by repeating that index once as a subscript and once as a superscript. The real functions $x^{d}$ of a real variable $d$ are defined and contianous over $d_{1} \leq d \leq d_{2}$, the norm is defined by

$$
\left\|x^{d}\right\|=\max _{d_{1} \leq d \leq d_{2}}\left|x^{d}\right|
$$

and the other operations are interpreted in the usual manner. We take the vector space $E_{1}$ to be also a space of continuous functions $V^{\star}$ defined over $\alpha_{1} \leq \alpha \leq \alpha_{2}$ with a norm defined by

$$
\left\|V^{x}\right\|=\max _{\alpha_{1} \leq \alpha \leq \alpha_{2}}\left|V^{x}\right|
$$

(1) Cf. the bibliography for finite dimensional differential geometry given in the introduction.

(2) A. D. Mrchal, "Amer. Journal of Math. ", loc. cit., and "Proc. of the Nationa] Academy of Sciences », 1930-1931 (four papers), loc. cit. 
A linear connection can be taken to be the funetional $\Gamma \times\left[x^{c}, V^{\beta}, \delta x^{b}\right]$ defined by (')

$$
\Gamma^{\alpha}\left[x^{d}, V^{\beta}, \delta x^{b}\right)=\Gamma_{\beta b}^{\alpha}\left[x^{d}\right] V^{\beta} \delta x^{b}+\Gamma_{b}^{a}\left[x^{d}\right] V^{\alpha} \delta x^{b},
$$

where $\Gamma_{\beta b}^{\alpha}\left[x^{d}\right]$ is a functional whose values are continuous functions of the three variables $\alpha, \beta, b$ and where $I_{b}^{\alpha}\left[x^{d}\right]$ is a functional whose values are continuous functions of the two variables $\alpha, b$.

The partial FRECHEx differential of the linear connection ean be taken to be in the normal form

$$
\left\{\begin{array}{c}
\Gamma^{\top}\left[\left[x^{d}, V^{\beta}, \delta_{1} x^{b} ; \delta_{2} x^{c}\right]=\left(\Gamma_{\beta b}^{\alpha}\left[x^{d}\right] V^{\beta}+{ }^{\prime} \Gamma_{b}^{\alpha}\left[x^{d}\right] V^{\alpha}\right) \delta_{1} x^{b} \delta_{2} x^{b}\right. \\
+\left(\Gamma_{\beta b, c}^{\alpha}\left[x^{d}\right] V^{\beta}+\Gamma_{b, \delta}^{\alpha}\left[x^{d}\right] V^{\alpha}\right) \delta_{1} x^{b} \delta_{2} x^{c},
\end{array}\right.
$$

where ${ }^{\prime} \Gamma_{\beta b}^{\alpha}\left[x^{d}\right], \Gamma_{b}^{\alpha}\left[x^{d}\right], \Gamma_{\beta b, c}^{\alpha}\left[x^{d}\right], \Gamma_{b, c}^{\alpha}\left[x^{d}\right]$ are four functionals of $x^{d}$ whose values are continuous functions of their respective free variables.

Clearly for any chosen positive numbers $a$ and $A$ there exist functionals of the form (2.1) and (2.2) such that all our postulates are satisfied by them for the given $a$ and $A$.

§ 3. The curvature form. - From the existence of the partial differential $\Gamma(x, V, y ; \delta x)$ follows the continuity in $x$ of $\Gamma(x, V, y)$ while from Postulates 2,3 , and 4 follows the continuity of $\Gamma(x, V, y)$ in $V$ and $y$ separately. Hence the theorem.

THeorem 3.1. - The linear connection $\Gamma(\mathrm{x}, \mathrm{V}, \mathrm{y})$ is continuous jointly in the three variables $\mathrm{x}, \mathrm{V}, \mathrm{y}$ and is a bilinear function of $\mathrm{V}$ and $\mathrm{y}$.

From the continuity of $\Gamma(x, V, y ; \delta x)$ in $x$ and this theorem we have.

Theorey 3.2. - The partial differential $\mathrm{I}(\mathrm{x}, \mathrm{V}, \mathrm{y} ; \delta \mathrm{x})$ is continuous in the set $\mathrm{x}, \mathrm{V}, \mathrm{y}, \delta \mathrm{x}$ and is a trilinear function of $\mathrm{V}, \mathrm{y}$ and $\delta \mathrm{x}$.

Definition of $B\left(x, V, \delta_{1} x, \delta_{2} x\right)$. - A function $\left({ }^{2}\right) \mathrm{B}\left(\mathrm{x}, \mathrm{V}, \delta_{1} \mathrm{x}, \delta_{2} \mathrm{x}\right)$, the curvature (form), is defined by

$$
\begin{gathered}
B\left(x, V, \delta_{1} x, \delta_{2} x\right)=\Gamma\left(x, V, \delta_{1} x ; \delta_{2} x\right)-\Gamma\left(x, V, \delta_{2} x ; \delta_{1} x\right) \\
+\Gamma\left(x, \Gamma\left(x, V, \delta_{1} x\right), \delta_{2} x\right)-\Gamma\left(x, \Gamma\left(x, V, \delta_{2} x\right), \delta_{1} x\right)
\end{gathered}
$$

in terms of the postulated linear connection $\Gamma\left(\mathrm{x}, \mathrm{V}, \hat{o}_{1} \mathrm{x}\right)$ and its partial differential $\Gamma\left(\mathrm{x}, \mathrm{V}, \delta_{1} \mathrm{x} ; \delta_{2} \mathrm{x}\right)$.

(1) In this instance, the variables $b, c, d, e, f$ are real variables ranging over the closed interval $\left(d_{1}, d_{2}\right)$, and $\alpha, \beta, \gamma$ are real variables ranging over the closed interval $\left(\alpha_{1}, \alpha_{2}\right)$.

(2) We remind the reader of the convention about the range of the arguments of functions. Here, of course, $x$ ranges over the region $\left(x_{0}\right)_{a}$. 
THEOREM 3.3. - The curvature $\mathrm{B}\left(\mathrm{x}, \mathrm{V}, \delta_{1} \mathrm{x}, \delta_{2} \mathrm{x}\right)$ defined in (3.1) is continuous in the set $\mathrm{x}, \mathrm{V}, \delta_{\mathrm{t}} \mathrm{x}, \delta_{\mathrm{t}} \mathrm{x}$. Moreover it is a trilinear function of $\mathrm{V}$, $\delta_{1} \mathrm{x}$ and $\delta_{2} \mathrm{x}$, and satisfies the identity

$$
B\left(x, V, \delta_{1} x, \delta_{2} x\right)=-B\left(x, V, \delta_{z} x, \delta_{1} x\right) .
$$

We are thus led to the following fundamontal theorem, whose proof can be obtained by a slight modification of the proof of a more general existence theorem on total differential equations in abstract vector spaces (').

Theorem 3.4. - If

$$
B\left(x, V, \delta_{1} x, \delta_{2} x\right) \equiv 0
$$

then there exists one and only one function $\xi(x)$ that enjoys the following properties:

a) $\xi(x)$ satisfies the total differential equation

$$
\xi(x ; \delta x)=-\Gamma(x, \xi(x), \delta x) ;
$$

b) $\xi(\mathrm{x})$ takes on the arbitrarily given initial value $\xi_{0}$ for $\mathrm{x}=\mathrm{x}_{0}$;

c) the first differential of $\xi(\mathrm{x})$ exists and is given ly (3.4);

d) the second differential of $\xi(\mathrm{x})$ exists, is symmetric in $\delta_{1} \mathrm{x}$ and $\delta_{3} \mathrm{x}$, and is given explicitly by

$$
\xi\left(x ; \delta_{1} x ; \delta_{2} x\right)=\Gamma\left(x, \Gamma\left(x, \xi(x), \delta_{2} x\right), \delta_{1} x\right)-\Gamma\left(x, \xi(x), \delta_{1} x ; \delta_{2} x\right) .
$$

In the last theorem the initial parameter $\xi_{0}$ is an arbitrarily chosen element in the vector space $E_{1}$ while $x_{n}$ is a fixed element of $E$. To exhibit the dependence of the solution function $\xi(x)$ on the initial abstract parameters $x_{0}$ and $\xi_{a}$, let us write $\xi\left(x, x_{0}, \xi_{0}\right)$ in the place of $\xi(x)$. We shall prove.

Theorem 3.5. - The function $\xi\left(\mathrm{x}, \mathrm{x}_{0}, \alpha\right)$ of Theorem 3.4 is a linear function of $\alpha$.

Proof: It can be shown that

$$
\xi\left(x, x_{0}, \alpha\right)=\lim _{n \rightarrow \infty} \xi_{n}\left(x, x_{0}, \alpha\right),
$$

where $\dot{\xi}_{0}=\alpha$ and

$$
\xi_{n+1}\left(x, x_{0}, \alpha\right)=\alpha-\int_{0}^{1} \Gamma\left(x_{0}+s\left(x-x_{0}\right), \xi_{n}\left(x_{0}+s\left(x-x_{0}\right), x_{\mathrm{n}}, \alpha\right), x-x_{0}\right) d s,
$$

the integral (*) being in the sense of KERNER-Graves.

(1) A. D. Mrohal and V. Elconin, loc. cit.

( ${ }^{2}$ L. Graves, "Trans. of Am. Math. Soc.", vol. 29 (1927); M. Korner, "Prace Mr* tematyczno-Fizyczne », vol. XI (1932). 
On making use of the continnity properties of the linear connection $T$ and employing a complete induction on (3.7) we find that for each $n \xi_{n}\left(x, x_{0}, \alpha\right)$ is a linear function of $x$. But $\lim _{n \rightarrow \infty} \xi_{n}\left(x, x_{0}, \alpha\right)$ exists equal $10 \xi\left(x, x_{0}, \alpha\right)$. Hence an application of a theorem of BANACH (1) on the linearity of the limit function of a convergent sequence of linear functions shows the validity of our theorem.

Both Theorem 3.4 and Theorem 3.5 are local theorems in the sense that the initial parameter $\infty_{0}$ of the vector space $E$ is fixed and is not an arbitrarily chosen element from some fixed set of elements. The following theorem is a theorem " in the large » and follows without much difficulty from another general existence theorem $\left({ }^{2}\right)$ of MichaL and Euconis.

Theorem 3.5. - Under the hypothesis of Theorem 3.4 there exists a convex region ( $\left.{ }^{3}\right) \mathrm{W}$ in $\mathrm{E}\left(\left(\mathrm{x}_{0}\right)_{a}\right)$ such that the conclusions of Theorem 3.4 and Theorem 3.5 hold for all $\mathrm{x}$ in $\mathrm{W}$ and for an arbitrarily chosen element $\overline{\mathrm{x}}$ in $\mathrm{W}$ instead of the fixed element $\mathrm{x}_{n}$.

THEOREM 3.6. - If there exists a solution $\xi(\mathrm{x})$ of the total differential equation

then

$$
\xi(x ; \delta x)=-\Gamma(x, \xi(x), \delta x)
$$

$$
B\left(x, \xi(x), \delta_{1} x, \delta_{2} x\right) \equiv 0
$$

in $\mathrm{x}, \delta_{1} \mathrm{x}, \delta_{2} \mathrm{x}$.

Proof: For such a $\xi(x)$, the second differential $\xi\left(x ; \delta_{1} x ; \delta_{2} x\right)$ is given by (3.5). By the postulates and previous theorems we see that $\xi\left(x ; \delta_{3} x ; \delta_{2} x\right)$ is continuous in $x$. Hence by a theorem of KenNer ( $\left.{ }^{+}\right)$on the symmetry of second FRÉCHET differentials, we have

$$
\xi\left(x ; \delta_{1} x ; \delta_{2} x\right)=\xi\left(x ; \delta_{2} x ; \delta_{1} x\right) \text {. }
$$

This however is condition (3.8) for the given $\xi(x)$.

THEOREM 3.7. - If there exists a fanily of solutions $\mathrm{F}(\mathrm{x}, \alpha)$, linear in $\alpha$ ( $x$ ranging over a vector space, say $\mathrm{E}_{2}$ ), of the total differential equation

$$
\xi(x ; \delta x)=-\Gamma(x, \xi(x), \delta x)
$$

such that $\mathrm{F}(\mathrm{x}, x)$ is a solvable linear function (with $\overline{\mathrm{F}}(\mathrm{x}, \mathrm{V})$, a function

(1) S. Banaon, « Fundamenta Math., loc. eit.

(2) A. D. Mrohal and V. Erconin, loc. eit.

( $\left.{ }^{3}\right)$ A region $W$ is convex if $x, y \varepsilon W$ implies that $t x+(1-t) y \in W$ for $0 \leq t \leq 1$.

(4) M. Kerner, "Annals of Math. ", loc. cit. 
on $\mathrm{E}\left(\left(\mathrm{x}_{0}\right)_{\mathrm{a}}\right) \mathrm{E}_{1}$ to $\mathrm{E}_{2}$, its inverse function), then

$$
B\left(x, V, \delta_{1} x, \delta_{2} x\right) \equiv 0
$$

in $\mathrm{x}, \mathrm{V}, \delta_{1} \mathrm{x}$ and $\delta_{z} \mathrm{x}$, and the linear connection can be expressed in the form

$$
\Gamma(x, V, \delta x)=-F(x, \vec{F}(x, V) ; \delta x),
$$

where $\mathrm{F}(\mathrm{x}, \alpha ; \delta \mathrm{x})$ is a partial differential of $\mathrm{F}(\mathrm{x}, \alpha)$.

Proof: By hypothesis we have

$$
F(x, \alpha ; \delta x)=-\Gamma(x, F(x, \alpha), \delta x) .
$$

Hence by an application of Theorem 3.6 we see that

$$
B\left(x, F(x, \alpha), \delta_{1} x, \delta_{2} x\right) \equiv 0
$$

in $x, \alpha, \delta_{1} x, \delta_{2} x$. From the arbitrariness of the parameter $\alpha$, it follows that we may take

$$
\alpha=\bar{F}(x, V),
$$

where $\bar{F}(x, V)$ is the inverse function to the solvable linear function $F(x, \alpha)$. Hence from identities of type (1.6) and from (3.12) we obtain the desired condition (3.9) of the theorem. Finally on substituting in (3.11) the expression for $\alpha$ as given by (3.13) we obtain the expression (3.10) for the linear connection.

COROLLARY: Under the hypotheses of Theorem 3.7 the linear connection $\Gamma(\mathrm{x}, \mathrm{V}, \delta \mathrm{x})$ can also be written in the form

$$
\Gamma(x, V, \delta x)=F(x, \bar{F}(x, V ; \delta x)),
$$

where $\overline{\mathrm{F}}(\mathrm{x}, \mathrm{V} ; \delta \mathrm{x})$ is a partial differential of $\overline{\mathrm{F}}(\mathrm{x}, \mathrm{V})$, the inverse funclion to the solvable linear function $\mathrm{F}(\mathrm{x}, \alpha)$.

Proof: From the fundamental identities (1.6) we have

$$
F(x, \bar{F}(x, V))=V .
$$

With the aid of Theorem 1.1, Theorem 1.4 and a theorem of FRECHET (') on the differentials of functions of funotions, we obtain the formula

$$
F(x, \vec{F}(x, V) ; \delta x)+F(x, \bar{F}(x, V ; \ddot{z} x))=0 .
$$

The expression (3.14) for the linear connection follows readily from (3.16) and the relation (3.10) of Theorem 3.7. The result (3.14) also follows directly from (3.15) and (3.7).

(1) M. Fréchet, "Annales Sc. Ee. Normale Sup. ", loc. cit. 
Before we continue the abstract theory in the next paragraph, it would be instructive to write down the curvature form for the functional instance of the postulates. We retain here the same notations used in the brief treatment at the end of $\S 2$. Clearly

$$
\Gamma^{\alpha}\left[x^{b}, \Gamma^{\beta}\left[x^{d}, V \curlyvee, \delta_{1} x^{b}\right], \delta_{2} x^{c}\right]=\left(\lambda_{r^{b c}}^{\alpha} V^{\Upsilon}+\lambda_{b c}^{\alpha} V^{\alpha}\right) \delta_{1} x^{b} \delta_{2} x^{c}
$$

where

$$
\left\{\begin{array}{l}
\lambda_{\gamma b c}^{\alpha}=\Gamma_{\gamma b}^{\beta} \Gamma_{\beta c}^{\alpha}+\Gamma_{b}^{(\gamma)} \Gamma_{\gamma^{c}}^{\alpha}+\Gamma_{\gamma^{b}}^{\alpha} \Gamma_{c}^{\alpha}, \\
\lambda_{b c}^{\alpha}=\Gamma_{b}^{\alpha} \Gamma_{c}^{\alpha},
\end{array}\right.
$$

and the parenthesis around $\gamma$ in $\Gamma_{b}^{(\gamma)}$ denotes that the variable $\gamma$ is an exception to the integration convention and is thus a free variable.

We thus have the curvature form of our functional instance

$$
B^{\alpha}\left[x^{d}, V^{\beta}, \delta_{1} x^{b}, \delta_{2} x^{c}\right]=\left(\omega_{\beta b c}^{\alpha} V^{\beta}+\omega_{b e}^{\alpha} V^{\alpha}\right) \delta_{1} x^{b} \delta_{z} x^{c},
$$

where the functionals $\omega_{\beta b c}^{x}$ and $\omega_{b c}^{\alpha}$ of $x^{d}$ are given respectively by

and

$$
\left\{\begin{array}{c}
\omega_{\beta b c}^{\alpha}=\Gamma_{\rho b, c}^{\alpha}-\Gamma_{\beta c, b}^{\alpha}+\Gamma_{\beta b}^{\alpha} \Gamma_{\gamma c}^{\alpha}-\Gamma_{\beta c}^{\alpha} \Gamma_{\gamma b}^{\alpha}+\Gamma_{b}^{(\beta)} \Gamma_{\beta c}^{\alpha} \\
-\Gamma_{c}^{(\beta)} \Gamma_{\beta b}^{\alpha}+\Gamma_{\beta b}^{\alpha} \Gamma_{c}^{\alpha}-\Gamma_{\beta c}^{\alpha} \Gamma_{b}^{\alpha}
\end{array}\right.
$$

$$
\omega_{b c}^{\alpha}=\Gamma_{b, c}^{\alpha}-\Gamma_{c, b}^{\alpha} .
$$

\& 4. Additional theorems on linear connections and curvature. - In this paragraph we give a few theorems (Theorems 4.1 and 4.3 ) that make use of the postulates for a linear connection given in paragraph 2 as well as some new postulates that are put in the form of hypotheses of theorems. The hypotheses of Theorem 4.2 below do not employ the given postulates of paragraph 2.

THEOREM 4.1. - Let $\mathrm{E}_{2}$ be a vector space, distinct or not from the vector spaces $\mathrm{E}$ and $\mathrm{E}_{1}$ of the postulates. Let $\mathrm{G}(\mathrm{x}, \alpha)$ be a function on $\mathrm{E}\left(\left(\mathrm{x}_{u}\right)_{\mathrm{a}}\right) \mathrm{E}_{2}$ to $\mathrm{E}_{1}$ that enjoys the following properties:

1) $\mathrm{G}(\mathrm{x}, \alpha)$, for each $\alpha$ of $\mathrm{E}_{2}$, satisfies the total differential equation

$$
\xi(x ; \delta x)=-\Gamma(x, \xi(x), \delta x)
$$

2) $\mathrm{G}(\mathrm{x}, \alpha)$ is a solvable linear function of $\alpha$. Let $\overline{\mathrm{G}}(\mathrm{x}, \beta)$, a function on $\mathrm{E}\left(\left(\mathrm{x}_{0}\right)_{2}\right) \mathrm{E}_{1}$ to $\mathrm{E}_{2}$, be its inverse function.

Then the function $\mathrm{F}\left(\mathrm{x}, \xi_{0}\right)$ defined by

$$
F\left(x, \xi_{0}\right)=G\left(x, \bar{G}\left(x_{0}, \xi_{0}\right)\right)
$$

is the unique solution of the total equation (4.1) that takes on the initial 
value $\xi_{0}$ for $\mathrm{x}=\mathrm{x}_{0}$. Furthermore $\mathrm{F}\left(\mathrm{x}, \xi_{0}\right)$ is a solvable linear function with inverse function $\overline{\mathrm{F}}\left(\mathrm{x}, \xi_{0}\right)$ given by

$$
\bar{F}\left(x, \xi_{0}\right)=G\left(x_{0}, \bar{G}\left(x, \xi_{0}\right)\right)
$$

Proof: Obviously $F\left(x, \xi_{0}\right)$ satisfies the total equation (4.1) and has the property

$$
F\left(x_{0}, \xi_{0}\right)=\xi_{0}
$$

An application of Theorem 3.7 shows that $B\left(x, V, \delta_{1} x, \delta_{2} x\right) \equiv 0$. Hence by Theorem 3.4 , the function $F\left(x, \xi_{0}\right)$ is the unique solution with the property (4.4).

From the fundamental relations (1.6) for solvable linear functions and from our hypotheses on $G(x, \infty)$ we obtain by an evident calculation

$$
F\left(x, \bar{F}\left(x, \xi_{0}\right)\right)=\xi_{0}=\bar{F}\left(x, F\left(x, \xi_{0}\right)\right) .
$$

This completes the proof of the theorem.

The next theorem does not make use of the postulates for a linear connection.

Theorem 4.2. - Let $\mathrm{E}, \mathrm{E}_{1}, \mathrm{E}_{2}$ be three vector spaces. Let $\mathrm{F}(\mathrm{x}, \alpha)$ be a solvable linear function on $\mathrm{E}\left(\left(\mathrm{x}_{n}\right)_{b}\right) \mathrm{E}_{2}$ to $\mathrm{E}_{1}$ with $\overline{\mathrm{F}}(\mathrm{x}, \beta)$, a function on $\mathrm{E}\left(\left(\mathrm{x}_{0}\right)_{b}\right) \mathrm{E}_{1}$ to $\mathrm{E}_{2}$, as its inverse function. Further assume that the first partial differential $\mathrm{F}\left(\mathrm{x}, \alpha ; \delta_{1} \mathrm{x}\right)$ exists; and that the second partial differential $\mathrm{F}(\mathrm{x}, \alpha$; $\left.\delta_{1} \mathrm{x} ; \delta_{2} \mathrm{x}\right)$ exists continuous in $\mathrm{x}$. Then there exist two functions $\Delta(\mathrm{x}, \mathrm{V}, \delta \mathrm{x})$ and $\mathrm{B}_{\Delta}\left(\mathrm{x}, \nabla, \delta_{1} \mathrm{x}, \delta_{2} \mathrm{x}\right)$ defined by

and

$$
\Delta(x, V, \delta x)=F(x, \bar{F}(x, V ; \delta x))
$$

$$
\left\{\begin{array}{c}
B_{\Delta}\left(x, V, \delta_{1} x, \delta_{2} x\right)=\Delta\left(x, V, \delta_{1} x ; \delta_{2} x\right)-\Delta\left(x, V, \delta_{2} x ; \delta_{1} x\right) \\
+\Delta\left(x, \Delta\left(x, V, \delta_{1} x\right), \delta_{2} x\right)-\Delta\left(x, \Delta\left(x, V, \delta_{2} x\right), \delta_{1} x\right)
\end{array}\right.
$$

respectively. Moreover

$$
B_{\Delta}\left(x, \quad V, \delta_{1} x, \delta_{2} x\right) \equiv 0 .
$$

Proof: With the aid of Theorem 1.4 we see that $\bar{F}(x, V ; \delta x)$ exists and hence the function $\Delta(x, V, \delta x)$ is well defined. The function $\Delta(x, V, \delta x)$ can also be written in the form

$$
\Delta(x, V, \delta x)=-F(x, \vec{F}(x, V) ; \delta x) .
$$

On making special use of the theorems on FRÉHET differentials developed in $\$ 1$ one can show without much diffieulty that the first partial dif- 
ferential $\Delta\left(x, V, \delta_{1} x ; \delta_{2} x\right)$ of $\Delta\left(x, V, \delta_{1} x\right)$ exists, is a trilinear function of $V$, $\delta_{1} x$ and $\delta_{2} x$, is continuous in the set $x, V, \delta_{1} x, \delta_{2} x$, and is given by

$$
\begin{aligned}
\Delta\left(x, V, \delta_{1} x ; \delta_{2} x\right)= & -F\left(x, \bar{F}(x, V) ; \delta_{1} x ; \delta_{2} x\right) \\
& -F\left(x, \bar{F}\left(x, V ; \delta_{2} x\right) ; \delta_{1} x\right) .
\end{aligned}
$$

Hence $B_{\Delta}\left(x, V, \delta_{1} x, \delta_{2} x\right)$ is well defined, is a trilinear function of $V, \delta_{1} x$ and $\delta_{2} x$, and is continuous in the set $x, V, \delta_{1} x, \delta_{2} x$.

From the relation

$$
\bar{F}\left(x, \Delta\left(x, V, \delta_{1} x\right)\right)=\bar{F}\left(x, V ; \delta_{1} x\right)
$$

we obtain by an evident calculation the result

$$
\Delta\left(x, \Delta\left(x, V, \delta_{1} x\right), \delta_{2} x\right)=-F\left(x, \bar{F}\left(x, V ; \delta_{1} x\right) ; \delta_{2} x\right) .
$$

We have however the symmetry relation

$$
F\left(x, \bar{F}(x, V) ; \delta_{1} x ; \delta_{2} x\right)=F\left(x, \bar{F}(x, V) ; \delta_{2} x ; \delta_{1} x\right) .
$$

The identity (4.7) follows now readily from (4.6), (4.9), (4.11) and (4.12).

CoRoLlary: Under the hypotheses of Theorem 4.2 there exist two positive numbers $\mathrm{A}$ and $\mathrm{a} \leq \mathrm{b}$ with respect to which the function $\Delta(\mathrm{x}, \mathrm{V}, \delta \mathrm{x})$ satisfies the postulates for a linear connection $\Gamma(\mathrm{x}, \mathrm{V}, \mathrm{ox})$.

The vector spaces $E_{1}$ and $E$ considered in the postulates may or may not be identical. Onder both circumstances the curvature $B(x, V, y, z)$ based on the linear connection $\Gamma(x, V, y)$ was seen to satisfy the identity

$$
B(x, V, y, z)=-B(x, V, z, y) .
$$

In case the vector space $E$ is identical with $E_{1}$, the sum

$$
B(x, U, V, W)+B(x, W, U, V)+B(x, V, W, U)
$$

is well defined, and the important question arises as to the form of the connection $\Gamma$ in order that this cyclical sum vanish. It follows immediately from Theorem 4.2 and its corollary that this cyclical sum vanishes when the connection is the $\Delta(x, V, \delta x)$ of the theorem and the vector spaces $E$ and $E_{1}$ are identical.

If the vector spaces $E$ and $E_{1}$ are distinct, the symmetry of the connection $\Gamma(x, V, y)$ in $V$ and $y$ has no meaning. However if the vector spaces are identical and $\Gamma(x, U, V)$ is symmetric in $U$ and $V$, then the partial differential $\Gamma(x, U, V ; W)$ is also symmetric in $U$ and $V$. Hence by direct calculation we obtain : 
THEOREM 4.3. - If the vector space $\mathrm{E}$ of the postulates is identical with $\mathrm{E}_{\mathrm{r}}$ and if the connection $\Gamma(\mathrm{x}, \mathrm{U}, \mathrm{V})$ is symmetric in $\mathrm{U}$ and $\mathrm{V}$, then the curvature form $\mathrm{B}(\mathrm{x}, \mathrm{U}, \mathrm{V}, \mathrm{W})$ based on this symmetric linear connection satisfies the cyclical identity

$$
B(x, U, V, W)+B(x, W, U, V)+B(x, V, W, U)=0 .
$$

$\$ 5$. Definition of parallelism with respect to a curve. - Let $x(t)$ be a function whose argument is a real variable $t$ and whose values are in the vector space $E$. We assume $x(t)$ to have the following properties:

1) $x(t)$ is defined and admits a continuous derivative (in the norm) for $t$ in $t_{0} \leq t \leq t_{1}$.

2) $x\left(t_{0}\right)=x_{0}$, where $x_{0}$ is the element of $E$ given in the postulates.

3) $\left\|x(t)-x_{0}\right\|<a$ for all $t$ in $\left(t_{0}, t_{1}\right)$.

Clearly there always exists such functions $x(t)$.

The set of values of the function $x(t)$ as $t$ ranges over the interval $\left(t_{0}, t_{1}\right)$ will be called a «nrve» with $x\left(t_{0}\right)$ and $x\left(t_{1}\right)$ as its end points and parameterized with a parameter $t$. The same curve can be parameterized with a different parameter $s$ by the usual (considered in classical geometry) parameter transformation $s=f(t)$, where $f(t)$ is a real valued increasing function of $t$. We observe that a curve as defined above lies wholly in a convex region of the vector space $E$.

Consider now the abstract differential equation

$$
\frac{d V}{d t}=-\Gamma\left(x, V, \frac{d x}{d t}\right)
$$

where $y=x(t)$ is a given curve and $\Gamma$ our postulated linear connection. Let

$$
\mathbf{Q}(t, V) \equiv-\mathrm{\Gamma}\left(x(t), V, \frac{d x(t)}{d t}\right)
$$

then the above differential equation can be written in the form

$$
\frac{d V}{d t}=\mathbf{Q}(t, V)
$$

where $Q(t, V)$ is linear in $V$. Since $\Omega(t, V)$ is continuous in $t$ for $t$ in $\left(t_{0}, t_{1}\right)$ it follows that $Q(t, V)$ is continuous in the set $t, V$. Therefore the hypotheses of a known existence theorem (I) are satisfied and we have the theorem:

(1) M. KmRner, "Prace Matematyczno-Fizyczne», loc. cit. 
THEOREM. - If $\mathrm{y}=\mathrm{x}(\mathrm{t})$ is the equation of a given curve described above, then there exists a number $\overline{\mathrm{t}} \leq \mathrm{t}_{1}$ such that for $\mathrm{t}$ in $\mathrm{t}_{\mathrm{i}} \leq \mathrm{t}<\overline{\mathrm{t}}$ the differential equation

$$
\frac{d V}{d t}=-\Gamma\left(x, V, \frac{d x}{d t}\right)
$$

has one and only one solution $\mathrm{V}(\mathrm{t})$, with continuous derivative $\frac{\mathrm{dV}}{\mathrm{dt}}$, that takes on the initial value $V_{0}$ for $t=t_{0}$.

Thus at each point $x(t)$ of the given curve for $t_{0} \leq t<\bar{t}$ there is associated a unique element, "contravariant» vector $V(t)$, whenever $V\left(t_{n}\right)$ is arbitrarily given. $V(t)$ may be called a parallel vector field along the given curve. Hence for any chosen $t^{\prime}$ in $t_{0} \leq t<\bar{t}$, there exists a unique contra. variant » vector $V\left(t^{\prime}\right)$, which we may call the vector parallel to $V\left(t_{0}\right)$ with respect to the given curve and localized at the point $t^{\prime}$ on the curve.

\$6. A set of postulates with a normed rectorial ring. - We consider in this paragraph a new set of postulates that involve a normed vectorial ring and a class of operations called contractions. This leads to a class of abstract «Ricei » forms. The author has been led to this class of objects by this studies pertaining to infinitely dimensional functional geometries.

We take a vector space $E_{1}$ and consider another associated manifold $E_{2}$ that we call a normed vectorial ring with a class of contractions. By this we mean a vector space $E_{2}$ (with elements $\sigma, \tau, \Phi, \psi, \omega, \omega_{1}, \omega_{2}, \omega_{3} \ldots$ ) that satisfies the following postulates (1).

Postulate A. There exists a bilinear function $\Phi\left(\omega_{1}, \omega_{2}\right)$ on $E_{2}^{z}$ to $E_{2}$.

Postulate B. $\Phi\left(\Phi\left(\omega_{1}, \omega_{2}\right), \omega_{3}\right)=\Phi\left(\omega_{1}, \Phi\left(\omega_{2}, \omega_{3}\right)\right)$, the associativity condition.

Postulate C. There exists a non-empty class of linear functions $\{C(\omega)\}$ on $E_{2}$ to $R$.

Postulate D. $C\left(\Phi\left(\omega_{1}, \omega_{2}\right)\right)=C\left(\Phi\left(\omega_{2}, \omega_{2}\right)\right)$.

Postulate E. There exists a bilinear function $f(\omega, V)$ on $E_{2} E_{1}$ to $E_{1}$ such that $f(\omega, V)=0$ for all $V$ in $E_{1}$ implies $\omega=0$.

Postulate F. $f\left(\Phi\left(\omega_{1}, \omega_{2}\right), V\right)=f\left(\omega_{1}, f\left(\omega_{2}, V\right)\right)$.

Finally we postulate:

Postulate $G$. There exists a function $\omega(x, V)$ on $E_{4}\left(\left(x_{0}\right)_{b}\right) E_{1}$ to $E_{2}$, linear in $V$.

Postulate H. The first partial differential $\omega(x, V ; \delta x)$ exists and is continuous in $x$.

(1) An independent set of postulates will require long cireumlocutions and a lengthy investigation. 
Postulate I. $f(\omega(x, V), \delta x)=f(\omega(x, \delta x), V)$.

Postulate $J$. There exists a function $\psi(x, V, \delta x)$ on $\left.E_{1}\left(x_{0}\right)_{b}\right) E_{1}^{2}$ to $E_{z}$, continuous separately in $x$ and in $\delta x$.

Postulate K. $f\left(\psi\left(x, V, \delta_{1} x\right), \delta_{2} x\right)=f\left(\omega\left(x, V ; \delta_{2} x\right), \delta_{1} x\right)$.

For convenience of notation we write the function $\Phi\left(\omega_{1}, \omega_{2}\right)$ simply as $\omega_{1} \omega_{3}$ and the function $f(\omega, V)$ simply as $\omega V$. It is elear from postulate $F$ that $f\left(\Phi\left(\omega_{1}, \omega_{2}\right), V\right)$ can be written as $\omega_{1} \omega_{2} V$ without any ambiguity.

THEOREM. - There exist two positive numbers A and $\mathrm{a} \leq b$ such that the symmetric linear connection defined by

$$
\Gamma(x, V, \delta x)=\omega(x, V) \delta x
$$

satisfies the six postulates for a linear connection given in section 2 if in these postulates the vector space $\mathrm{E}$ is taken identical to $\mathrm{E}_{1}$. Moreover $\Psi(x, V, \delta x)$ is bilinear in $\mathrm{V}$ and $\delta \mathrm{x}$, and

$$
\begin{aligned}
& \Gamma(x, V, \delta x)=\Gamma(x, \delta x, V) \\
& \psi(x, V, \delta x)=\psi(x, \delta x, V) .
\end{aligned}
$$

In terms of this simplified notation, the curvature form based on the present symmetric connection (6.1) can be written as follows after a few rearrangements

$$
\begin{gathered}
B\left(\left(x, V, \delta_{1} x, \delta_{2} x\right)=\left(\psi\left(x, V, \delta_{1} x\right)-\omega\left(x, V ; \delta_{1} x\right)+\omega\left(x, \omega(x, V) \delta_{1} x\right)\right.\right. \\
\left.-\omega\left(x, \delta_{1} x\right) \omega(x, V)\right) \delta_{2} x .
\end{gathered}
$$

Thus to each contraction function $C(n)$ of the class $\{C(\omega)$,$\} postulated$ in Postulate $\mathrm{C}$ there corresponds an abstract « Ricei » form given by

$$
C\left(\psi\left(x, V, \delta_{1} x\right)-\omega\left(x, V ; \delta_{1} x\right)+\omega\left(x, \omega(x, V) \hat{z}_{1} x\right)-\omega\left(x, \delta_{1} x\right) \omega(x, V)\right) .
$$

To show the consistency of the set of postulates consisting of the vector space postulates and the postulates $A-K$, we shall give an instance arising from the author's ( $\left.{ }^{1}\right)$ studies on functional differential geometries. One of the interesting features of this instance is found in the existence of a class of contraction functions $\{C(\omega)\}$ consisting of an infinite number of contraction functions $C(\omega)$. There thus exists an infinite set of "Ricei $»$ forms. It is of interest to note that in the well known finite dimensional differential geo* metries there exists only one contraction within a multiplicative constant.

We take the vector space $E$ (vector space $E_{1}$ is taken identical to $E$ ) to be the «space of continuous functions $x^{a} \gg$ considered in $\$ 2$. For the

(1) A. D. Michal, loc. cit. 
normed vectorial ring $E_{2}$ with a class of contractions we take the set of ordered pairs of continuous functions $\left(\omega_{c}^{b}, \omega^{d}\right)$ with the following interpretation of the elements and operations. Let

$$
\sigma \equiv\left(\sigma_{c}^{b}, \sigma^{d}\right), \quad \tau \equiv\left(\tau_{e}^{b}, \tau^{d}\right),
$$

where $\sigma_{c}^{b}$ and $\tau_{c}^{b}$ are continuous on the square $d_{1} \leq b, c \leq d_{2}$, and $\sigma^{d}$ and $\tau^{d}$ are continaous on the interval $\left(d_{1}, d_{2}\right)$. Take

$$
\begin{aligned}
& \sigma+\tau \equiv\left(\sigma_{c}^{b}+\tau_{c}^{b}, \sigma^{d}+-\tau^{d}\right), \text { addition of elements; } \\
& r \sigma \equiv\left(r \sigma_{c}^{b}, r \sigma^{d}\right), \text { multiplication by real numbers } r ; \\
& \Phi(\sigma, \tau) \equiv\left(\sigma_{e}^{b} \tau_{c}^{e}+\sigma^{b} \tau_{c}^{b}+\sigma_{c}^{b} \tau^{(c)}, \sigma^{d} \tau^{d}\right) ; \\
& \|\sigma\| \equiv\left(d_{2}-d_{1}\right) \max _{b, c}\left|\sigma_{c}^{b}\right|+\max _{d}\left|\sigma^{d}\right| ; \\
& f(\sigma, V) \equiv \sigma_{c}^{b} V^{c}+\sigma^{b} V^{b} ; \quad \text { or } \sigma_{b}^{b_{1}}, \text { where } b_{1} \text { is any chosen number in }\left(d_{1}, d_{2}\right) \text {, or } \\
& C(\sigma) \equiv \sigma_{b}^{b} \text { or } \\
& \sigma_{b}^{b}+\int_{d_{1}}^{d_{9}} \sigma^{b} d b \text {, etc. }
\end{aligned}
$$

Next we take for $x^{e}$ in $\max _{e}\left|x^{e}-x_{0}^{e}\right|<a$

$$
\omega(x, V) \equiv\left(\omega_{o}^{b}[x, V], \quad \omega^{d}[x, V]\right)
$$

where $\omega_{c}^{b}[x, V]$ and $\omega^{d}[x, V]$ are functionals of the continuous funetions $x^{e}$ and $V f$, and are given respectively by

$$
\omega_{\mathrm{b}}^{b}[x, V]=\Gamma_{d c}^{b}[x] V^{a}+\Gamma_{* 0}^{b}[x] V^{b}+\Gamma_{c_{*}}^{b}[x] V^{(c)}
$$

and

$$
\omega^{a}[x, V]=\Gamma_{b}^{d}[x] V^{b}+\Gamma^{d}[x] V^{d} .
$$

The values of the five functionals $\Gamma$ in $(6.5)$ and (6.6) can be taken as continuous functions of all their respective free variables.

To satisfy the symmetry postulate $I$ we restrict two of the functionals $\Gamma$ to satisfy the relations

$$
\Gamma_{d e}^{b}[x]=\Gamma_{e d}^{b}[x], \quad \Gamma_{* e c}^{b}[x]=\Gamma_{c}^{b}[x]
$$

By classical methods one can show that conditions (6.7) are necessary and sufficient for the symmetry of our linear connection. Our symmetric linear connection $\Gamma(x, V, \delta x)$ ean be written as follows

$$
\Gamma_{c d l}^{b}[x] V^{c} \delta x^{d}+\Gamma_{c_{*}}^{b}[x] V^{c} \delta x^{c}+\Gamma_{d}^{b}[x]\left(V^{c} \delta x^{b}+V^{b} \delta x^{c}\right)+\Gamma^{b}[x] V^{b} \delta x^{b} .
$$

Finally we have to define a $\psi(x, V, \delta x)$ function for our instance. To do this we need the expression for the partial differential $\omega(x, V ; \delta x)$. We restrict 
the functionals $(6.5)$ and $(6.6)$ so that $\omega(x, V ; \delta x)$ exists for our instance and is given explicitly by

$$
\left\{\begin{array}{c}
\omega(x, V ; \delta x) \equiv\left(\omega_{e, d}^{b}[x, V] \delta x^{d}+{ }^{b} \omega_{e}^{b}[x, V] \delta x^{b}+{ }_{c} \omega_{c}^{b}[x, V] \delta x^{(c)}\right. \\
\left.\omega_{, d}^{b}[x, V] \delta x^{a}+{ }^{b} \omega^{b}[x, V] \delta x^{b}\right)
\end{array}\right.
$$

where the values of the five functionals $\omega$ are assumed to be continuous functions of all their respective free variables. We note howerer that the continuous indices written on the left of an $\omega$ functional do not indicate an additional argument for the values of the functional but are merely used as a notational device to denote the various «types of functional derivatives». For example, ${ }^{b} \omega_{c}^{b}[x, V]$ is a functional whose values are continuous funetions of two variables $b$ and $c$ and not necessarily continuous functions of three variables with two arguments filled in with the same letter.

We now take

$$
\left\{\begin{array}{c}
\Psi(x, V, \delta x) \equiv\left(\omega_{c, d}^{b}[x, V] \delta x^{c}+{ }_{d} \omega_{d}^{b}[x, V] \delta x^{(t)}+\omega_{, d}^{b}[x, V] \delta x^{b}\right. \\
\left.{ }^{b} \omega_{c}^{b}[x, V] \delta x^{(c)}+{ }^{b} \omega^{b}[x, V] \delta x^{b}\right) .
\end{array}\right.
$$

\$ 7. A serond linear connection and curvature form. - In this section we shall deal with the consequences of new postulates in addition to the six postulates of $\S 2$ and the postulates for the vector spaces $E$ and $E_{1}$. Besides the vector spaces $E$ and $E_{1}$ we shall be concerned with still another associated manifold, a vector space $E_{2}$. The elements of this new vector space $E_{2}$ may habitually be called "covariant vectors». It should be noted howerer that in some of the instances the elements of $E_{2}$ may be contravariant vectors.

Thronghout the present paragraph, unless otherwise stated, we shall use the letters $L, M, \Lambda, \eta, \theta, \ldots$ with or without subscripts, for elements of $E_{z}$. All the other letters used will be in accordance with the conventions layed down for the notations of $\& 2$.

Postulate 7. There exists a function $\Lambda(x, \eta, y)$, a second linear connection, on $\left.E\left(x_{0}\right)_{a}\right) E_{2} E$ to $E_{2}$.

Postulate 8. $\Lambda\left(x, \eta_{1}+\eta_{2}, y\right)=\Lambda\left(x, \eta_{1}, y\right)+\Lambda\left(x, \eta_{2}, y\right)$.

Postulate 9. $\Lambda\left(x, \eta, y_{1}+y_{2}\right)=\Lambda\left(x, \eta, y_{1}\right)+\Lambda\left(x, \eta, y_{2}\right)$.

Postulate 10. $\|\Lambda(x, \eta, y)\|<B\|\eta\|\|y\|$, where $B$ is a fixed positive number.

Postulate 11. The first partial differential $\Lambda(x, \eta, y ; \delta x)$ exists.

Postulate 12. $\Lambda(x, \eta, y ; \delta x)$ is continuous in $x$.

Postulate 13. There exists a function $[\xi, \eta]$ on $E_{1} E_{2}$ to $R$.

Postulate 14. $\left[\xi_{1}+\xi_{2}, \eta\right]=\left[\xi_{1}, \eta\right]+\left[\xi_{2}, \eta\right]$.

Postulate 15. $\left[\xi, \eta_{1}+\eta_{2}\right]=\left[\xi, \eta_{1}\right]+\left[\xi, \eta_{2}\right]$. 
Postulate 16. $[\xi, \eta]$ is continnous in $\xi$.

Postulate 17. $[\xi, \eta]$ is continuous in $\eta$.

Postulate 18. If $[\xi, \eta]=0$ for all $\xi$ of $E_{1}$ then $\eta=0$.

Postulate 19. If $[\xi, \eta]=0$ for all $\eta$ of $E_{2}$ then $\xi=0$.

Postulate 20. $-[\Gamma(x, \xi, y), \eta]=[\xi, \Lambda(x, \eta, y)]$.

The functional instance presented in paragraph two for the consistency of the eight postulates for the first linear connection can be used to show the consistency of Postulates 1.20 together with the postulates for the three vector spaces $E, E_{1}$ and $E_{2}$.

The first linear connection given in (2.1) is

$$
\Gamma_{\beta b}^{\alpha}\left[x^{d}\right] \xi \beta \delta x^{b}+\Gamma_{b}^{\alpha}\left[x^{d}\right] \xi_{\delta}^{\alpha} \delta x^{b} .
$$

It is clear how one can restrict the functionals $\Gamma_{\beta b}^{\alpha}\left[x^{d}\right]$ and $\Gamma_{b}^{g}\left[x^{d}\right]$ so that

$$
\Gamma_{\beta b}^{\alpha}\left[x^{d}\right] \eta_{x} \delta x^{b}+\Gamma_{b}^{(\beta)}\left[x^{\alpha}\right] \eta_{p} \delta x^{b}
$$

would satisfy the postulates for the second linear connection $\Lambda(x, \eta, \delta x)$. We further interpret $[\xi, \eta]$ as $\xi \eta_{\alpha}$. In this instance we interpret the vector space to be a «space of continuous functions $\eta_{\alpha}$ » defined over the interval $\left(\alpha_{1}, \alpha_{2}\right)$. Clearly Postulates 18 and 19 are satisfied by virtue of the fundamental lemma of the calculus of variations. Postulate 20 is obviously satisfied and all the remaining postulates can be satisfied by making evident restrictions on the functionals $\Gamma_{\beta b}^{\alpha}\left[x^{d}\right]$ and $\Gamma_{b}^{\beta}\left[x^{a}\right]$.

Definition of second curvature form $\left({ }^{1}\right) \mathrm{L}\left(\mathrm{x}, \eta, \delta_{1} \mathrm{x} ; \delta_{2} \mathrm{x}\right)$

$$
\begin{aligned}
& L\left(x, \eta, \delta_{1} x, \delta_{2} x\right)=\Lambda\left(x, \eta, \delta_{1} x ; \delta_{2} x\right)-\Lambda\left(x, \eta, \delta_{2} x ; \delta_{1} x\right) \\
& \quad+\Lambda\left(x, \Lambda\left(x, \eta, \delta_{2} x\right), \delta_{1} x\right)-\Lambda\left(x, \Lambda\left(x, \eta, \delta_{1} x\right), \delta_{2} x\right),
\end{aligned}
$$

a function defined on $E\left(\left(x_{0}\right)_{a}\right) E_{2} E^{2}$ to $E_{2}$.

The whole content of paragraphs $2,3,4$ and 5 can by obvious modifications be made to hold good for the second linear connection. We leave the details for the reader. For example, the correspondent of Theorem 3.3 is

Theовем 7.1 - The second curvature $\mathrm{L}\left(\mathrm{x}, \eta, \delta_{1} \mathrm{x}, \delta_{3} \mathrm{x}\right)$ defined in (7.3) is continuous in the set $\mathrm{x}, \eta, \delta_{1} \mathrm{x}, \delta_{2} \mathrm{x}$. Moreover it is a trilinear function of $\eta$, $\delta_{1} \mathrm{x}$ and $\delta_{2} \mathrm{x}$, and satisfies the identity

$$
L\left(x, \eta, \delta_{1} x, \delta_{2} x\right)=-L\left(x, \eta, \delta_{2} x, \delta_{1} x\right) .
$$

There is a simple and fundamental relation between the two curvature

(1) Compare third and fourth term with the third and fourth term respectively of $B\left(x, \xi, \delta_{1} x, \delta_{2} x\right)$. Furthermore $B\left(x, \xi, \delta_{1} x, \delta_{2} x\right)$ corresponds to integrability conditions $\delta_{1} \delta_{2} \xi-\delta_{2} \delta_{1} \xi=0$ while $L$ corresponds to integrability conditions $\delta_{2} \delta_{1} \eta-\delta_{1} \delta_{2} \eta=0$. 
forms $B\left(x, \xi, \delta_{1} x, \delta_{2} x\right)$ and $L\left(x, \eta, \delta_{1} x, \delta_{2} x\right)$. We embody this result in the following theorem.

THEOREM 7.2 - The two curvature forms $\mathrm{B}\left(\mathrm{x}, \xi, \delta_{1} \mathrm{x}, \delta_{2} \mathrm{x}\right)$ and $\mathrm{L}\left(\mathrm{x}, \eta, \delta_{1} \mathrm{x}, \delta_{2} \mathrm{x}\right)$ are related by the following identity

$$
\left[B\left(x, \xi, \delta_{1} x, \delta_{2} x\right), \eta\right]=\left[\xi, L\left(x, \eta, \delta_{1} x, \delta_{2} x\right)\right] .
$$

Proof: On taking partial differentials of

we obtain

$$
\left[\Gamma\left(x, \xi, \delta_{1} x\right), \eta\right]=\left[\xi, \Lambda\left(x, \eta, \delta_{1} x\right)\right]
$$

The result (7.6) is justified on making use of the postulates and the well known theorem that «differentiable functions of differentiable functions possess differentials $»$.

From (7.5) we obtain

$$
\left[\Gamma\left(x, \Gamma\left(x, \xi, \delta_{1} x\right), \delta_{2} x\right), \eta\right]=\left[\Gamma\left(x, \xi, \delta_{1} x\right), \Lambda\left(x, \eta, \delta_{2} x\right)\right] .
$$

Again by employing (7.5) on the right hand side of (7.7) we find that

$$
\left[\Gamma\left(x, \Gamma\left(x, \xi, \delta_{1} x\right), \delta_{2} x\right), \eta\right]=\left[\xi, \Lambda\left(x, \Lambda\left(x, \eta, \delta_{2} x\right), \delta_{1} x\right)\right] .
$$

The nse of (7.6) and (7.8) in an evident calculation proves the theorem.

Corollary: $A$ necessary and sufficient condition that $\mathrm{B}\left(\mathrm{x}, \xi, \delta_{1} \mathrm{x}, \delta_{2} \mathrm{x}\right)$ vanish identically is that $\mathrm{L}\left(\mathrm{x}, \eta, \delta_{1} \mathrm{x}, \delta_{2} \mathrm{x}\right)$ vanish identically.

DeFinition. - The linear function of $\delta \mathrm{x}$

$$
\xi(x ; \delta x)+\Gamma(x, \xi(x), \delta x)
$$

may be called the «covariant» differential of the «contravariant» vector field $\xi(x)$ while the linear function of $\delta x$

$$
\eta(x ; \delta x)-\Lambda(x, \eta(x), \delta x)
$$

may be called the «covariant» differential of the « covariant » vector field $\eta(x)$. Let

$$
\xi(x: \delta x) \equiv \xi(x ; \delta x)+\Gamma(x, \xi, \delta x)
$$

and

$$
\eta(x: \delta x) \equiv \eta(x ; \delta x)-\Lambda(x, \eta, \delta x) .
$$

Theorem 7.3 - If $\mathrm{f}(\mathrm{x})$ is defined by

$$
f(x)=[\xi(x), \eta(x)]
$$

and if $\xi(\mathrm{x})$ and $\eta(\mathrm{x})$ possess first differentials, then

$$
f(x ; \delta x)=[\xi(x ; \delta x), \eta(x)]+[\xi(x), \eta(x: \delta x)] .
$$


Proof:

$[\xi(x ; \delta x), \eta(x)]=[\xi(x ; \delta x), \eta(x)]+[\Gamma(x, \xi(x), \delta x), \eta(x)]$.

Hence a special use of Postulate 20 yields the result

$[\xi(x ; \delta x), \eta(x)]=[\xi(x ; \delta x), \eta(x)]+[\xi(x), \Lambda(x, \eta(x), \delta x)]$.

Clearly

$$
[\xi(x), \eta(x: \delta x)]=[\xi(x), \eta(x ; \delta x)]-[\xi(x), \Delta(x, \eta(x), \delta x)] .
$$

The theorem is now immediate from this and the preceding relation. 